\title{
Bortezomib-Induced Blepharitis: A Case Report
}

ISSN: 2578-0379

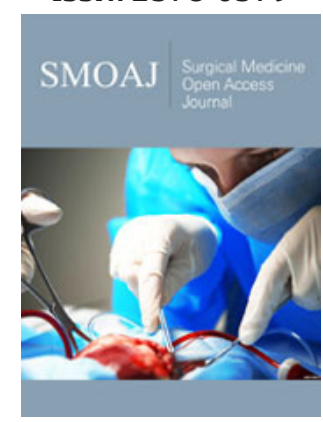

*Corresponding author: Kamal AlZahran, Department of Hematology-Oncology, Middle East Institute of Health (MEIH), Beirut, Lebanon.

Submission: 眮 January 16, 2020

Published: 眥January 27, 2020

Volume 3 - Issue 3

How to cite this article: Ahmad K, Pamela S, Antoine A A, Kamal A, Elise Slim, et al. Bortezomib-Induced Blepharitis: A Case Report. Surg Med Open Acc J.3(3). SMOAJ.000561.2020. DOI: 10.31031 /SMOAJ.2020.03.000561.

Copyright@ Kamal AlZahran, This article is distributed under the terms of the Creative Commons Attribution 4.0 International License, which permits unrestricted use and redistribution provided that the original author and source are credited.

\author{
Ahmad Khalil ${ }^{1}$, Pamela Sfeir ${ }^{1}$, Antoine Abi Abboud ${ }^{2}$, Kamal AlZahran ${ }^{1 *}$, Elise $^{2}$ \\ Slim$^{3}$ and Evelyne Helou ${ }^{4}$ \\ ${ }^{1}$ Department of Hematology-Oncology, Lebanon \\ ${ }^{2}$ Department of Gastro-Enterology, Lebanon \\ ${ }^{3}$ Department of Ophthalmology, Lebanon \\ ${ }^{4}$ Department of Hematology-Oncology, Lebanon
}

\begin{abstract}
Introduction: Bortezomib is a proteasome inhibitor approved for the treatment of multiple myeloma and has known manageable toxicities. Blepharitis is an inflammatory condition of the eyelid that leads to formation of chalazia both causing visual field disturbance. Bortezomib induced blepharitis has not been well reported in the literature.
\end{abstract}

Case: we present a case of 76-year-old lady with multiple myeloma who developed bortezomibinduced blepharitis and chalazia. Patient was successfully treated after topical ocular therapy, systemic antibiotherapy and omission of bortezomib.

Keywords: Multiple myeloma; Blepharitis; Chalazia; Bortezomib

\section{Introduction}

Multiple myeloma is characterized by a neoplastic proliferation of plasma cells in the bone marrow producing a monoclonal immunoglobulin and resulting in extensive skeletal destruction. One of the used treatment regimens for MM being the VCD protocol (Velcade ${ }^{\circledR}$ or Bortezomib, Cyclophosphamide and Dexamethasone) [1,2]. Bortezomib is a proteasome inhibitor that acts by disrupting the cell cycle and inducing apoptosis, usually tolerated in the outpatient settings with manageable toxicities, the most common ones being peripheral neuropathy and thrombocytopenia [1,2]. Blepharitis is an inflammatory condition of the eyelid margin causing ocular and visual discomfort leading to formation of chalazia (lipogranulomatous lesions) [3,4]. It is classified as anterior blepharitis when it involves the eyelid skin and the follicules and posterior when it involves the Meibomian glands; it is usually associated with staphylococcus aureus colonization, infestation with parasites, Meibomian gland dysfunction, rosacea, systemic use of docetaxel $[3,4]$. Bortezomib has been associated with ocular complications but it has not been well characterized. We herein present a case of Bortezomib-induced blepharitis that has been successfully treated.

\section{Case Report}

A 76-year-old lady diagnosed with multiple myeloma IgG kappa light chain stage III in August 2018 after she was found to have anemia, elevated creatinine $(1.5 \mathrm{~g} / \mathrm{dL})$ and a serum protein electrophoresis showing $17.5 \mathrm{~g} / \mathrm{dL}$ of $\mathrm{M}$ protein (monoclonal peak). Patient was started on VCD protocol (weekly based) and zoledronic acid for malignant hypercalcemia. After completion of two treatment cycles, in October 2018, patient was found to have a left painful itchy red eye. Ophthalmologic exam showed edema and redness of the left upper eyelid with preserved visual fields, no diplopia, no discharge. Fundoscopy was normal. There was no evidence of viral or upper respiratory tract infection. Patient then started on doxycycline (daily dose of 100mg) and local framycetin (Frakidex®) to apply four times daily on the affected eye while continuing her chemotherapy treatment. She then developed 2 new upper eyelid chalazia, while receiving her blepharitis treatment. Significant improvement was observed when combining blepharitis treatment with bortezomib cessation. In 
November 2018, patient had a right trans-cervical hip fracture, no anemia, stable chronic kidney disease and negative PET scan so chemotherapy was stopped. Follow up ophthalmologic exam after 3 months showed a complete remission of the blepharitis (Figure 1).

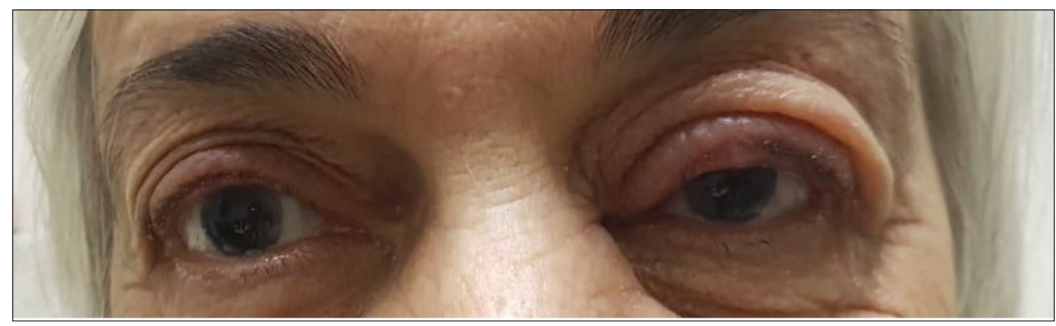

Figure 1: Left red eye and chalazia in left upper eyelid.

\section{Discussion}

We reported a case of bortezomib induced blepharitis and chalazia successfully treated after topical therapy and systemic antibiotherapy (doxycycline) associated with omission of chemotherapy. A literature review done showed 28 cases of bortezomib induced blepharitis reported. Most recently, the association between bortezomib and chalazia was classified as a "possible" adverse drug reaction (ADR) based on the World Health Organization's definition of ADRs and an analysis of both case reports in the literature as well as reports submitted to the National Registry of Drug-Induced Ocular Side Effects [5,6].

In a study conducted by Sklar et al. [7]16 patients treated with bortezomib developed ocular complications after 3.4 months in average. $100 \%$ of blepharitis and $44 \%$ of chalazia resolved completely after ocular therapy alone whereas $80 \%$ of chalazia and $60 \%$ of blepharitis resolved after ocular therapy and chemotherapy omission with longer periods needed for remission of blepharitis (117 day) versus chalazia (74 days) [7]. In our case, after a treatment with oral doxycycline and topical framycetin, blepharitis and chalazia didn't improve. It only resolved after 30 days when bortezomib was omitted.

Grob SR et al. [8]. reported a case series in which 6 patients developed chalazia within 3.3 months of bortezomib therapy beginning. All conservative measures (including warm compresses and lid hygiene) failed. Four patients underwent incision and curettage and 5 of 6 patients had bortezomib suspended or discontinued due to eye discomfort [8]. Ince bortezomib inhibits the ubiquitin proteasome pathway, proapoptotic molecules accumulates leading to apoptosis of neoplastic cells. In parallel, bortezomib influence other inflammatory pathways including NF-kB, JAK/STAT, and MAP kinase, promoting release of proinflammatory cytokines. Interference of all these pathways in the eyelids contributes in inflammatory flares causing blepharitis and/ or chalazia [9]. So, the pathogenesis of blepharitis and chalazia in proteasome inhibitor treated patients is postulated to be related to inflammation [9-11]. This hypothesis is further proved by the fact that blepharitis resolved with local anti-inflammatory treatment and systemic antibiotic.

Studies postulated that a baseline eye examination is encouraged before starting proteasome inhibitor therapy [7]. Treatment of chalazia/blepharitis induced by bortezomib should be started with conservative measures (hot compresses in combination with at least 1 topical antibiotic and/or steroid drop, and possible oral antibiotics as well). If eyelid complications persist after this treatment, omission of bortezomib should be considered with a switch to alternative proteasome inhibitors (carfilzomib or ixazomib). Oral doxycycline can be added if no improvement. After bortezomib omission, if eyelid complications resolve, are challenge can be considered. However, if eyelid complications recur upon bortezomib re challenge, the drug should be discontinued again [7]. In our case, patient improved after local therapy, oral doxycycline and omission of bortezomib. No re-challenge or switch to another proteasome inhibitor was done in our case because of the patient's fracture and stable disease.

\section{Conclusion}

We herein presented a case of bortezomib-induced blepharitis and chalazia that has been successfully treated after ocular therapy, systemic antibiotherapy and omission of the bortezomib. So, awareness, early detection, and prompt management of proteasome inhibitors eyelid complications, (although rare) will help improve the quality of life of patients.

\section{References}

1. Richardson PG, Barlogie B, Berenson J, Singhal S, Jagannath S, et al. (2003) A phase 2 study of bortezomib in relapsed, refractory myeloma. N Engl J Med 348(26): 2609-2617.

2. Jagannath S, Durie BG, Wolf J, Camacho E, Irwin D, et al. (2005) Bortezomib therapy alone and in combination with dexamethasone for previously untreated symptomatic multiple myeloma. $\mathrm{Br} \mathrm{J}$ Haematol 129(6): 776-783.

3. Kanski JJ (1994) Clinical ophthalmology: A systematic approach. $\left(5^{\text {th }}\right.$ edn), Butterworth-Heinemann Ltd, UK.

4. Ballen PH (1964) Inflammations of the lid. Int Ophthalmol Clin 4: 5-20.

5. Fraunfelder FW, Yang HK (2016) Association between bortezomib therapy and eyelid chalazia. JAMA Ophthalmol 134(1): 88-90.

6. Veys MC, Delforge M, Mombaerts I (2016) Treatment with doxycycline for severe bortezomib-associated blepharitis. Clin Lymphoma Myeloma Leuk 16(7): e109-e112.

7. Sklar AB, Gervasio KA, Leng S, Ghosh A, Chari A, et al. (2019) Management and outcomes of proteasome inhibitor associated chalazia and blepharitis: A case series. BMC Ophthalmology 19(1): 110.

8. Grob SR, Jakobiec FA, Rashid A, Yoon MK (2014) Chalazia associated with bortezomib therapy for multiple myeloma. Ophthalmology 121(9): 1845-1847. 
9. Sunwoo JB, Chen Z, Dong G, Yeh N, Crowl BC, et al. (2001) Nove proteasome inhibitor PS-341 inhibits activation of nuclear factorkappa B, cell survival, tumor growth, and angiogenesis in squamous cell carcinoma. Clin Cancer Res 7(5): 1419-1428.

10. Yun C, Mukhi N, Kremer V, Shinder R, Verma V, et al. (2015) Chalazia development in multiple myeloma: A new complication associated with bortezomib therapy. Hematol Rep 7(2): 5729.
11. Mohamed R, Massa H, Schwarz C, Grandjean HN, Samii K (2015) Bortezomib induced multiple chalazia: A case report. Case Reports in Clinical Medicine 4(1): 32-35.

For possible submissions Click below: 